\title{
La revista Medicina y Seguridad en el Trabajo y el respeto al derecho de autoría
}

\author{
Medicina y Seguridad del Trabajo journal and protecting copyright
}

\section{Jorge Veiga de Cabo}

Redactor Jefe de Medicina y Seguridad del Trabajo. Madrid. España.

\author{
Correspondencia \\ Jorge Veiga de Cabo \\ Instituto de Salud Carlos III \\ Escuela Nacional de Medicina del Trabajo \\ Severo Ochoa, s/n. P.8 Ciudad Universitaria \\ 28040 Madrid. España \\ Tfno: 918224031 \\ E-mail: revista.enm+@isciii.es
}

Resumen

El autor recuerda los principios básicos internacionales que amparan los derechos de autor aprobados por el Convenio de Berna para la Protección de las Obras Literarias y Artística, la Declaración Universal de Derechos Humanos y el Derecho de la Unión Europea del Tratado de Maastricht. Se analizan las circunstancias generales que amparan la autoría, y en particular en el marco de la biomedicina y de las ciencias de la Salud, así como las principales características del mercado editorial y los cambios que se han producido en los últimos años. Se describen los principales factores asociados a la aparición de la iniciativa Open Access y su compromiso con la preservación del Copyright y los derechos de autor. Se presenta la licencia Creative Commons como alternativa eficaz en la gestión de los derechos de autoría. Por último, se declara la adhesión oficial de la revista Medicina y Seguridad del Trabajo a la licencia Creative Commons bajo modalidad Reconocimiento -NoComercial - SinObraDerivada (by-nc-nd).

Palabras Clave: Derechos Humanos, derechos de autor, autoría, Copyright, acceso abierto, Open Access, CreativeCommons.

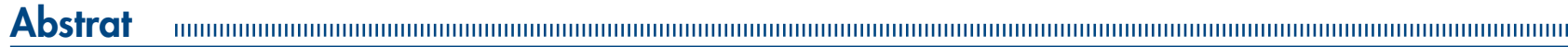

The author reviews the international basic principles of Copyrights approved by the Berne Convention for the Protection of Literary and Artistic Works, the Universal Declaration of Human Rights and the Treaty of Maastricht on the European Union Right. General circumstances to protect Copyrights are analyzed; in particular in Biomedicine and Health Sciences fields, as well as some other specific characteristics related with the publishing market and the main changes suffered in those fields throughout the years. This document describes the relevant factors associated with the emergence of the Open Access initiative and its commitment protecting Copyright.Creative Commons is presented as an effective alternative to preserve Copyrights. Finally, the Medicina y Seguridad del Trabajo journal officially declares its adhesion to Creative Commons under the Attribution-NonCommercial-NoDerivs (by-nc-nd) license.
\end{abstract}

Key Words: Human Rights, Copyright, authorship, Open Access, Creative Commons. 
El derecho de autor es uno de los principios básicos establecidos en la Declaración Universal de Derechos Humanos (DUDH) aprobado por la Asamblea General de las Naciones Unidas el 10 de diciembre de 1948 en París [Resolución 217 A (III)] y tiene por base el Convenio de Berna para la Protección de las Obras Literarias y Artísticas (más conocido como Tratado de Berna), firmado el 9 de septiembre de 1886 en Berna (Suiza) para los países de la Unión Europea y aquellos que voluntariamente quisieran adherirse. Ha sido revisado y actualizado en diferentes ocasiones, correspondiéndose la última al 28 de septiembre de 1979 y en la actualidad se encuentran adheridos 165 países.

Aunque el Tratado de Berna parece «a priori» referirse en exclusiva a las obras literarias y artísticas, en su Artículo 2 establece que «obras literarias y artísticas» comprende todas las producciones en el campo literario, científico y artístico, cualquiera que sea el modo o forma de expresión, tales como los libros, folletos y otros escritos, así como conferencias, alocuciones, sermones y otras obras de la misma naturaleza. Además establece un plazo mínimo de derechos de autoría durante los 50 años posteriores a la fecha de fallecimiento del autor.

A partir del Tratado de Maastricht, firmado en 1992 por los países que la integran, se establece el Derecho de la Unión Europea que contempla también una normativa referente a los derechos de autor, pero amplía el plazo mínimo de 50 años establecido por el Tratado de Berna a un periodo de vigencia de 70 años desde la muerte del autor.

Al margen de las legislaciones y normativas nacionales e internacionales vigentes, y en términos generales, los derechos de autor o "copyright» suelen encontrarse socialmente asumidos y reconocidos como un derecho moral inherente a la creación de cualquier obra cuando se trata de creaciones literarias o cualquier otra rama de lo que conocemos como expresiones artísticas (música, cine, pintura, artes gráficas, por citar algunas). Este concepto no se encuentra tan claramente asumido cuando nos encontramos frente a producciones científicas, y aún más dentro del área de la biomedicina o de las ciencias de la salud. En parte, esto se debe a que tradicionalmente y en la mayoría de los casos, las editoriales de este sector han controlado los derechos de autor (copyright) imponiendo la condición de cesión sus derechos a la editorial para que el artículo fuera publicado.

La aparición de Internet en 1986 revoluciona el escenario social y cultural del momento, surgiendo lo que hoy entendemos como sociedad digital o sociedad de la información. A partir de ese momento y sobre todo en la década de los 90, se producen una serie de fenómenos que promueven un cambio conceptual dentro de la sociedad y su forma de entender el acceso a la información como un derecho.

El principal cambio que se produce dentro del ámbito científico es que la edición electrónica se empieza a ver como un mecanismo eficaz, rápido, cómodo y expansivo de comunicar la ciencia, además de encontrarse exenta de costes adicionales, o siendo estos muy bajos. El imperio de la edición en papel comienza a tambalearse y se genera una enorme incertidumbre en los principales grupos editoriales que encuentran en esta situación una amenaza de sus intereses comerciales, si no son capaces de redefinir sus políticas editoriales y reconducir los procesos de comercialización de las suscripciones dentro de este nuevo escenario ante el que se encuentran.

La mayor parte de las editoriales optan por pasar de la edición en papel a la edición electrónica, inicialmente de forma compartida como mecanismo de cautela, y posteriormente de forma exclusiva.

Esta transformación del proceso editorial supuso también un cambio muy importante de las condiciones del sistema de contratación de suscripciones. Por una parte, se pasa de un modelo contractual en el que "se compra un producto tangible", como es la obtención de la publicación en papel por las biblioteca o particulares, a un "contrato de servicio de acceso a la edición electrónica" de las publicaciones. Esto significa que se cambia el contrato de compra por el que se recibe una revista científica cuyos contenidos se pueden consulta, almacenar e intercambiar en soporte papel, a un sistema de contrato de suscripción en línea que sólo permite obtener la licencia de acceso al archivo en formato electrónico durante el tiempo limitado que se haya establecido en las condiciones particulares del contrato, generalmente un año. Al finalizar este periodo, cualquier consulta o recuperación de algún documento de la base de datos tendrá unas condiciones impuestas por el grupo editorial. 
A finales de los 90, esta situación desencadena una situación de estrés y crisis dentro del sistema formal de la comunicación científica, como señala la Asociación de Bibliotecas Universitarias y de Investigación (Association of College and Research Libraries, ACRL). La creciente preocupación por parte de los grandes grupos editoriales por mantener el dominio y control del mercado dentro del nuevo escenario de la edición electrónica, genera una desproporcionada subida de precios de las suscripciones de las revistas científicas en su nueva versión de "licencia de acceso". De esta forma, para la gran mayoría de las bibliotecas y centros de documentación se hace difícil soportar los precios del mercado con sus presupuestos, lo que obliga a reducir drásticamente el número de suscripciones o a intentar asociarse en consorcios bibliotecarios para negociar mejores condiciones con las editoriales ${ }^{1}$.

Tal es este punto al que se llega, que a modo de ejemplo, en 2004 la Universidad de Harvard saca una nota en los Archivos de la Gaceta de la Universidad manifestando la posición que ha de tomar ante los incrementos sufridos por las suscripciones a las publicaciones ${ }^{2}$.

Esta situación genera una reacción por parte la comunidad científica, la cual toma conciencia de ser el motor que genera el material científico y que Internet puede ser una herramienta que ofrece alternativas eficaces para el acceso y la difusión de la información y el conocimiento. En paralelo se produce un proceso de concienciación similar entre las instituciones académicas, de investigación y administraciones públicas de los principales países promotores de la investigación, al darse cuenta de que se enfrentan a unas condiciones económicas de suscripción impuestas por el mercado editorial muy por encima de sus posibilidades, mientras son ellas, en su gran mayoría, las principales fuentes de financiación de la investigación y de la producción científica.

Finalmente se produce una convergencia de intereses profesionales e institucionales, surgiendo la iniciativa "Open Access o Acceso Abierto» como un movimiento tendente a promover el acceso libre y gratuito a la información científica respetando los principios éticos relacionados con la investigación y la publicación científica, manteniendo así como el compromiso de garantía de calidad de sus contenidos mediante la revisión por pares y otros sistemas de control de calidad. Además uno de los principios fundamentales de la Iniciativa Open Access, preservar la autoría y garantizar los derechos de copyright del autor sobre su obra ${ }^{3,4}$.

Aunque desde el punto de vista teórico preservar los derechos de autor pueda parecer algo sencillo, en la práctica supone cierta complejidad a la hora de abordar globalmente los derechos de autor en el escenario universal en el que se produce ciencia, tanto por la enorme diversidad de marcos legales con los que nos podemos encontrar en las procedencias de filiaciones de autores e instituciones, como por las distintas legislaciones de los diferentes países donde puedan encontrarse alojadas las múltiples plataformas de Acceso Abierto.

Además, a este mosaico legislativo habría que añadir las distintas opciones por las que cada autor pueda optar a la hora de ejercer los derechos de autoría en Acceso Abierto. Así, por ejemplo un autor puede querer preservar su derecho legítimo de copyright pero sólo en cuanto a los derechos comerciales sin renunciar a las ventajas que ofrece una amplia difusión de su obra mediante los sistemas y condiciones de acceso abierto sin fines comerciales, mientras otro puede querer mantener sólo el control de las obras derivadas de su obra original, pudiéndose presentar de esta forma, múltiples variedades de ejercer el derecho de autoría.

Una solución eficaz a este abanico de posibilidades se ofrece desde el modelo creado por "Creative Commons", una corporación sin ánimo de lucro, creada en 2001 por "Law School Stanford" y con recursos de "The Law School Center for Internet and Society" y que ofrece diferentes alternativas de ejercer dicho derecho ${ }^{6}$.

Creative Commons establece un sistema combinado de licencias que permiten decidir a los autores sobre las condiciones particulares del copyright, adaptándolo a los diferentes marcos legales de diferentes países, lo que podríamos entender como un "copyright a la carta». 
En el caso de España, la Universidad de Barcelona (UB) es la institución afiliada a Creative Commons y la que adaptó las combinaciones de licencias a la legislación vigente sobre propiedad intelectual del Estado Español, encontrándose públicamente disponibles en castellano y catalán desde el 1 de octubre de $2004^{7}$.

Básicamente existen cuatro condiciones de las licencias:

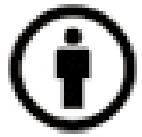

Reconocimiento (Attribution): En cualquier explotación de la obra autorizada por la licencia hará falta reconocer la autoría.

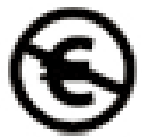

No Comercial (Non commercial): La explotación de la obra queda limitada a usos no comerciales.

Sin obras derivadas (No Derivate Works): La autorización para explotar la obra no incluye la transformación para crear una obra derivada.

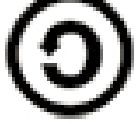

Compartir Igual (Share alike): La explotación autorizada incluye la creación de obras derivadas siempre que mantengan la misma licencia al ser divulgadas.

Con estas cuatro condiciones combinadas se pueden generar seis perfiles que recogen las opciones más frecuentes de ejercer los derechos de autoría:
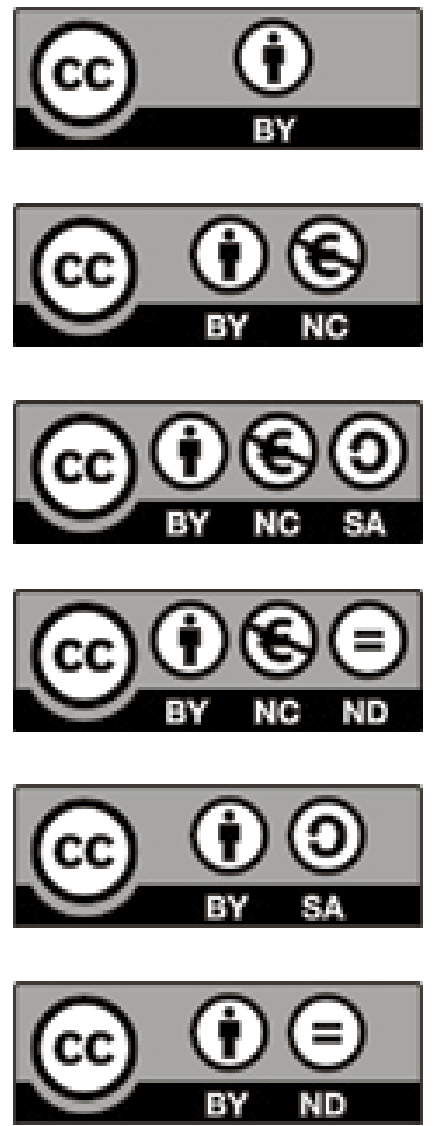

Reconocimiento (by): Se permite cualquier explotación de la obra, incluyendo una finalidad comercial, así como la creación de obras derivadas, la distribución de las cuales también está permitida sin ninguna restricción.

Reconocimiento - NoComercial (by-nc): Se permite la generación de obras derivadas siempre que no se haga un uso comercial. Tampoco se puede utilizar la obra original con finalidades comerciales.

Reconocimiento - NoComercial - CompartirIgual $(b y-n c$-sa): No se permite un uso comercial de la obra original ni de las posibles obras derivadas, la distribución de las cuales se debe hacer con una licencia igual a la que regula la obra original.

Reconocimiento - NoComercial - SinObraDerivada $(b y$-nc-nd): No se permite un uso comercial de la obra original ni la generación de obras derivadas.

Reconocimiento - Compartir Igual (by-sa): Se permite el uso comercial de la obra y de las posibles obras derivadas, la distribución de las cuales se debe hacer con una licencia igual a la que regula la obra original.

Reconocimiento - SinObraDerivada (by-nd): Se permite el uso comercial de la obra pero no la generación de obras derivadas.

La revista Medicina y Seguridad del Trabajo asumió el criterio de preservar los derechos de autoría en 2007 bajo la responsabilidad del actual equipo editorial. A partir del número 208 de ese mismo año, la revista incluye en las Normas de Publicación su declaración explícita de que se reserva exclusivamente los "derechos 
legales de reproducción del contenido y publicación en las bases de datos internacionales", lo que significa que no exige la cesión de los derechos de autor para la publicación de los artículos.

En ese mismo año la revista es aceptada en SciELO (Scientific Electronic Library Online) y en DOAJ (Directory of Open Access Journals), ${ }^{8}$ lo que supone un paso más en este sentido al aceptar de esta forma las condiciones de Open Access y el respeto a la autoría para difusión de publicaciones en sistema abierto.

A partir de junio de este año la revista se adhiere oficialmente a la licencia Creative Commons bajo la modalidad de Reconocimiento -NoComercial-SinObraDerivada ( $b y$ - $n \boldsymbol{c}$ - $n \boldsymbol{n}$ ), incorporando el correspondiente sello de adhesión a partir del número 227 de junio de 2012, el cual queda reflejado en las condiciones de Copyright de la revista que se encuentran en el apartado "sobre nosotros" de su espacio en SciELO así como en el de Redacción y Administración de sus formatos en PDF y e-Pub.

Este reconocimiento significa que los autores mantienen sus derechos de autoría y no permiten el uso comercial de la obra original ni de las posibles obras derivadas, la distribución de las cuales debe hacerse con una licencia igual a la que regula la obra original, respetando la autoría y referencia de la revista que debe ser siempre citada, así como permiten su divulgación mediante los sistemas de acceso abierto y la utilización de los contenidos por la comunidad científica internacional y el resto de la sociedad.

\section{REFERENCIAS BIBLIOGRÁFICAS}

1. Iniciativa ACRL para reformar la comunicación erudita. Disponible en: http://www.geotropico.org/1_2_documentos_acrl_ principios.html. (Consultado 28 de septiembre de 2012)

2. Harvard Gazette Archives [monograph on the Internet]. Cambridge (MA): The Harvard University; 2004. Disponible en: http:// www.news.harvard.edu/gazette/2004/02.05/10-libraries.html. (Consultado 28 de septiembre de 2012).

3. Sanz-Valero J, Veiga de Cabo J, Castiel LD. The Open Access Initiative in access to technical and scientific information in health sciences. RECIIS Electronic Journal of Communication, Information \& Innovation in Health. 2007;1:19-26. Disponible en: http:// www.reciis.cict.fiocruz.br/index.php/reciis/issue/view/9/showToc. (Consultado 28 de septiembre de 2012).

4. Sanz-Valero J, Castiel LD, Wanden-Berghe Lozano C, Juan Quilis V. Internet y la búsqueda de información en Salud Pública: desde la relevancia hacia la "revelancia". Gac Sanit. 2006;20:159-60.

5. Sanz-Valero Javier, D’Agostino Marcelo José, Castiel Luis David, Veiga de Cabo Jorge. La iniciativa Open Access, una visión de conjunto. Med. segur. trab. [Internet]. 2007 Jun;53(207):05-09.Disponible en:

6. http://scielo.isciii.es/scielo.php?script=sci_arttext\&pid=S0465-546X2007000200003\&lng=es.

7. http://dx.doi.org/10.4321/S0465-546X2007000200003. (Consultado 21 de septiembre de 2012).

8. Creative Commons. Disponible en: http://creativecommons.org (Consultado 21 de septiembre de 2012).

9. Creative Commons España. Disponible en: http://es.creativecommons.org/(Consultado 21 de septiembre de 2012 ).

10. Veiga de Cabo Jorge. Medicina y Seguridad del Trabajo ha superado los criterios de selección para integrarse en la Red SciELO (Scientific Electronic Library Online). Med. segur. trab. [Internet]. 2007 Jun [citado 2012 Oct 11] ; 53(207): 01-04. Disponible en: http://scielo.isciii.es/scielo.php?script=sci_arttext\&pid=S0465-546X2007000200002\&lng=es

11. http://dx.doi.org/10.4321/S0465-546X2007000200002. (Consultado 21 de septiembre de 2012). 